\title{
The Atherogenic Dyslipidemia Ratio Log (Tg)/Hdl-C Was Not Associated with Urinary Albumin Excretion Rate (Uaer) and Increased Cardiovascular Risk in Black Patients with Type 2 Diabetes
}

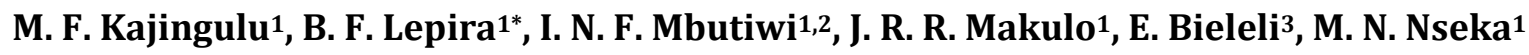 \\ ${ }^{1}$ Division of Nephrology, University of Kinshasa, Democratic Republic of Congo \\ ${ }^{2}$ Faculty of Medicine, University of Kikwit, Democratic Republic of Congo \\ ${ }^{3}$ Division of Diabetes and Metabolic Diseases, University of Kinshasa Hospital, University of Kinshasa, Democratic \\ Republic of Congo \\ Email: "Iepslepira@yahoo.fr
}

Received 23 December 2015; accepted 24 January 2016; published 27 January 2016

Copyright (C) 2016 by authors and Scientific Research Publishing Inc.

This work is licensed under the Creative Commons Attribution International License (CC BY).

http://creativecommons.org/licenses/by/4.0/

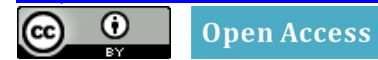

\begin{abstract}
Objective: The objective is to assess the relationship of Log (TG)/HDL-c as surrogate estimate of atherogenic dyslipidemia with urinary albumin excretion rate and cardiovascular risk among black patients with type 2 diabetes. Patients and methods: A post-hoc analysis of data from 181 type 2 diabetes patients enrolled in a cross-sectional study of urinary albumin excretion rate seen at a tertiary healthcare. Microalbuminuria and macroalbuminuria were defined as ACR 30 - 299.9 $\mathrm{mg} / \mathrm{g}$ and $A C R \geq 300 \mathrm{mg} / \mathrm{g}$, respectively. Quartiles of Log (TG)/HDL-c were used as surrogate estimates of atherogenic dyslipidemia. Cardiovascular risk was assessed using WHO chart for estimation of CV risk in low and middle income countries. Comparisons across Log (TG)/HDL-c quartiles were performed using one way ANOVA and Chi square for trend as appropriate. $P<0.05$ defined the level of statistical significance. Results: A high prevalence (69\%) of atherogenic dyslipidemia (AD) was observed in the present case series of Black Africans with type 2 diabetes. Average total cholesterol levels showed significant $(p=0.010)$ trends towards lower values across quartiles of Log (TG)/ HDL-c. No significant trends were observed for average UAER and cardiovascular risk across quartiles of Log (TG)/HDL-c. Conclusion: Log (TG)/HDL-c as a surrogate estimate of atherogenic failed to predict cardiovascular risk in the present case series of black patients with type 2 diabetes.
\end{abstract}

\footnotetext{
${ }^{*}$ Corresponding author.

How to cite this paper: Kajingulu, M.F., Lepira, M.F., Mbutiwi, I.N.F., Makulo, J.R.R., Bieleli, E. and Nseka, M.N. (2016) The Atherogenic Dyslipidemia Ratio Log (Tg)/Hdl-C Was Not Associated with Urinary Albumin Excretion Rate (Uaer) and Increased Cardiovascular Risk in Black Patients with Type 2 Diabetes. World Journal of Cardiovascular Diseases, 6, 14-20. http://dx.doi.org/10.4236/wjcd.2016.61003
} 


\section{Keywords}

\section{Log (TG)/HDL-C, Cardiovascular Risk, Type 2 Diabetes, Black Africans}

\section{Introduction}

Type 2 diabetes has now become an important public health problem in sub-Saharan Africa (SSA) and is associated with an increased cardiovascular (CV) morbidity and mortality attributed in part to the coexistence of other risk factors such as hypercholesterolemia [1]. Indeed, studies have consistently indicated that type 2 diabetes and dyslipidemia with mainly hypercholesterolemia frequently coexist and the risk of cardiovascular diseases associated with the coexistence of these two conditions is higher than the sum of the individual risks [1]. $\mathrm{CV}$ risk in type 2 diabetes is also increased in case of abnormal urinary albumin excretion rate (UAER), a marker of both kidney damage and extended endothelial dysfunction and reported to be associated with hypercholesterolemia [1]. Therefore, the early detection and treatment of hypercholesterolemia in type 2 diabetes patients particularly in case of abnormal UAER could help reducing CV morbidity and mortality [1].

Since mainly low-density lipoprotein cholesterol (LDL-c) is considered to play an important role in the dynamic process of atherogenesis [1]-[4], current guidelines recommend intensive lowering of LDL-c with statins in an attempt to reduce CV risk in type 2 diabetes [5] [6]. However, despite effective lowering of LDL-c at optimal or near-optimal levels [7] [8], residual risk of CVD remains still high in statin treated type 2 diabetes patients [2] [8]. This residual CV risk is thought to rely upon the presence of the so-called atherogenic dyslipidemia (AD), which is characterized by low high-density lipoprotein cholesterol (HDL-c) and/or high triglyceride (TG) levels [9]-[11]. An association between AD and abnormal UAER has been reported as well [11]-[13]. Therefore, type 2 diabetes patients with abnormal UAER could be at high risk for both atherogenic dyslipidemia and subsequent CVD. As a consequence, screening for atherogenic dyslipidemia in type 2 diabetes patients could help refining CV risk assessment and improve therapeutic decision making since AD responded better to fibrates than to statins [14]. In this regard, the ratio of fasting TG to fasting HDL-c [Log (TG)/HDL-c], with prior log transformation, could help to identify type 2 diabetes patients with or without abnormal UAE at high risk for CVD [14] [15]. Indeed, [log (TG)/HDL-c] has been reported to be associated with both microangiopathy and residual CV risk in Caucasian type 2 diabetes patients [15]-[17].

Aforementioned observations come mainly from studies on people of Caucasian origin [15] [16] [18]. Unfortunately, very few studies on AD in black type 2 diabetes patients with and without abnormal UAER are available. In sub-Sahara Africa, a study from Nigeria had found that diabetic patients had high levels of TG and low HDL-c [19]. In Democratic Republic of the Congo, the prevalence of abnormal UAER as micro or macroalbuminuria in diabetic patients has been reported to be of $29 \%$ and $19 \%$, respectively [20]. A previous study showed that type diabetes 2 patients with subclinical atherosclerosis had in average low HDL-c and high TG levels compared to those without atherosclerosis [21]. Unfortunately, the prevalence of AD as combined dyslipidemia was not evaluated. The aim of the present post hoc analysis was to evaluate the prevalence and severity of $\mathrm{AD}$ in black patients with type 2 diabetes using $\log (\mathrm{TG}) / \mathrm{HDL}-\mathrm{c}$ ratio as a surrogate marker of $\mathrm{AD}$ and to determine whether this ratio is associated with UAER and increased absolute CV risk.

\section{Patients and Methods}

To evaluate the relationship of Log (TG)/HDL-c ratio with UAER and cardiovascular risk, we performed a post-hoc analysis of data from 181 type 2 diabetes patients enrolled in a cross-sectional study of abnormal urinary albumin excretion (UAER) carried out at the University of Kinshasa Hospital from $1^{\text {st }}$ July to $30^{\text {th }}$ October, 2007. Data were obtained using a standard questionnaire which collected information on age, gender, duration of diabetes, smoking and alcohol use. Physical examination was performed in each patient to measure height, weight, waist circumference (WC), blood pressure and pulse rate; eye fundus examination was also obtained to search for diabetic retinopathy. The details of the study have already been described elsewhere [20]. In brief, UAER as urinary albumin to creatinine ratio (ACR) was determined in a morning spot urine using immunoassay method with an automatic device DCA Bayer $2000^{\circledR}$ (Bayer Health Care LLC, Indiana, USA). Normoalbuminuria, microalbuminuria and macroalbuminuria were defined as ACR < 30, $30-299$ and $\geq 300 \mathrm{mg} / \mathrm{g}$, respectively 
[22]. Fasting cholesterol and its subfractions as well as TG were determined using enzymatic methods with an automatic device Cholestech LDX ${ }^{\circledR}$ (Cholestech Corporation, USA). LDL-c was calculated using the Friedewald formula for patients with TG levels less than $400 \mathrm{mg} / \mathrm{dl}$ [23]. Non-HDL-c, as a surrogate marker of apolipoprotein B (Apo B), was calculated as total cholesterol minus HDL-c. For the present post-hoc analysis, AD was defined, according to 2013 International Consensus [24] as HDL-c $<40 \mathrm{mg} / \mathrm{dl}$ in men, $<50 \mathrm{mg} / \mathrm{dl}$ in women and/or triglycerides $\geq 150 \mathrm{mg} / \mathrm{dl}$. The continuous variable Log (TG)/HDL-c was used as a surrogate estimate of AD [15] [16] and the World Health Organization (WHO) chart for sub-Saharan Africa to estimate absolute CV risk [25].

\section{Statistical Analyses}

Baseline characteristics were summarized as means and SDs for continuous variables for normally distributed variables and median and range for skewed variables; categorical variables as relative frequencies in percent. Student t was used to compare means of two groups, as appropriate. Categorical variables were compared using Pearson's chi square, Fischer exact for trends as appropriate. Multiple regression analysis was used to identify determinants of absolute CV risk. P value $<0.05$ defined the level of statistical significance. All analyses were performed using the statistical software STATA version 10.1.

\section{Results}

Table 1 summarizes clinical characteristics of the study population according to quartiles of Log (TG)/HDL-c. One hundred eighty one type 2 diabetes patients, 53\% men, were included in the present post-hoc analysis. Their mean age was $56 \pm 11$ years with a median duration of diabetes of 4 (1 - 32) years. Smoking, hypertension and diabetic retinopathy were present in $8 \%, 77 \%$ and $80 \%$ of patients, respectively.

Biological characteristics according to quartiles of Log (TG)/HDL-c are depicted in Table 2. Average levels of glycemia, HbA1c, MDRD-GFR, atherogenic dyslipidemia and UAER were 165 (50 - 446) mg/dl, 9.0 (4.5 14.0) \%, 75 (2 - 234) $\mathrm{ml} / \mathrm{min} / 1.73 \mathrm{~m}^{2}, 69 \%$ and 30 (3 - 7800) $\mathrm{mg} / \mathrm{g}$, respectively. Average levels of lipids were $177 \pm 43 \mathrm{mg} / \mathrm{dl}, 117 \pm 40 \mathrm{mg} / \mathrm{dl}, 38 \pm 15 \mathrm{mg} / \mathrm{dl}, 139 \pm 40 \mathrm{mg} / \mathrm{dl}$ and 100 (45 - 650) mg/dl for TC, LDL-c, HDL-c, non-HDL-c and TG, respectively. Apart from TG and HDL-c levels as components of Log (TG)/HDL-c ratio, significant trends towards lower levels across quartiles of Log (TG)/HDL-c were observed for only TC; trends in other variables across quartiles of Log (TG)/HDL-c did not reach the level of statistical significance.

Table 1. Clinical characteristics of the study population according to quartiles of Log (TG)/HDL-c.

\begin{tabular}{|c|c|c|c|c|c|c|}
\hline & $\begin{array}{c}\text { All } \\
\mathrm{n}=181\end{array}$ & $\begin{array}{c}\text { Q1 } \\
\mathrm{n}=45\end{array}$ & $\begin{array}{c}\text { Q2 } \\
\mathrm{n}=45\end{array}$ & $\begin{array}{c}\text { Q3 } \\
\mathrm{n}=45\end{array}$ & $\begin{array}{c}\mathrm{Q} 4 \\
\mathrm{n}=46\end{array}$ & $\mathrm{p}$ \\
\hline Log (TG)/HDL-c & $0.064 \pm 0.031$ & $0.033 \pm 0.005$ & $0.048 \pm 0.004$ & $0.064 \pm 0.006$ & $0.108 \pm 0.025$ & - \\
\hline Age, yrs & $56 \pm 11$ & $56 \pm 10$ & $57 \pm 9$ & $56 \pm 12$ & $56 \pm 13$ & 0.961 \\
\hline Gender, M, \% & 53 & 56 & 42 & 47 & 43 & 0.580 \\
\hline $\mathrm{F}, \%$ & 47 & 44 & 58 & 53 & 57 & \\
\hline Diabetes duration, yrs & $4(1-32)$ & $5(1-32)$ & $5(1-25)$ & $3(1-25)$ & $4(1-23)$ & 0.478 \\
\hline Smoking, \% & 8 & 7 & 2 & 11 & 11 & 0.327 \\
\hline Alcohol intake, \% & 31 & 27 & 33 & 27 & 39 & 0.515 \\
\hline SBP, mm Hg & $141 \pm 25$ & $142 \pm 24$ & $144 \pm 23$ & $141 \pm 25$ & $136 \pm 26$ & 0.491 \\
\hline DBP, mm Hg & $87 \pm 13$ & $88 \pm 13$ & $86 \pm 11$ & $89 \pm 12$ & $85 \pm 14$ & 0.446 \\
\hline HR, b.min & $84 \pm 10$ & $85 \pm 13$ & $85 \pm 9$ & $84 \pm 11$ & $82 \pm 9$ & 0.600 \\
\hline BMI, $\mathrm{kg} / \mathrm{m}^{2}$ & $25.5 \pm 5.1$ & $25.3 \pm 4.3$ & $25.3 \pm 3.9$ & $25.4 \pm 5.6$ & $26.1 \pm 6.3$ & 0.841 \\
\hline $\mathrm{WC}, \mathrm{cm}$ & $90 \pm 12$ & $89 \pm 9$ & $90 \pm 12$ & $90 \pm 13$ & $93 \pm 14$ & 0.080 \\
\hline Retinopathy, \% & 80 & 80 & 76 & 82 & 80 & 0.881 \\
\hline
\end{tabular}

Data are expressed as mean \pm standard deviation, median and range or relative frequency in percent. Abbreviations: Log, logarithm; TG, triglycerides; HDL-c, high density lipoprotein cholesterol; Q, quartile; yrs, years; M, male; F, female; SBP, systolic blood pressure; DBP, diastolic blood pressure; HR, heart rate; BMI, body mass index; WC, waist circumference. 
Hypertension, global and abnormal obesity, atherogenic dyslipidemia and cardiovascular risk across quartiles of Log (TG)/HDL-c are depicted in Table 3. As expected, the prevalence of atherogenic dyslipidemia significantly ( $<$ 0.001) increased across Log (TG)/HDL-c quartiles (Table 3). However, trends in absolute CV riskacross quartiles of Log (TG)/HDL-c ratio did not reach the level of statistical significance.

\section{Discussion}

The main findings of the present post-hoc analysis are as follows. First, a high prevalence of AD was observed in the present case series of Black Africans with type 2 diabetes. Second, Log (TG)/HDL-c was not related to absolute CV risk. Third, mean levels of UAER did not increase across Log (TG)/HDL.

The high prevalence of AD in type 2 diabetes patients agrees with results of previous studies [11] [19] [26]. However, the prevalence of $69 \%$ in the present post-hoc analysis seems somewhat higher than that reported in other studies [16] [18]. This disparity can be explained by differences in criteria used to define the atherogenic dyslipidemia [15] [18] [24] and characteristics of studied populations.

Log (TG)/HDL-c was not significantly associated with absolute CV risk in the present post-hoc analysis. This finding does translate the current controversy around the ability of this ratio to accurately predict CV risk in Blacks of African descent with type 2 diabetes [27]. Indeed, If a previous study conducted in menopausal Nigerian women found Log (TG)/HDL-c to be an useful predictor of CV risk [28], a recent study of South and West African women [29] showed that this ratio was ineffective in this purpose. However, reports on white type 2 diabetes patients from Italy [30] and Belgium [15] [16] showed that Log (TG)/HDL-c was associated with

Table 2. Biological characteristics of the study population according to quartiles of Log (TG)/HDL-C.

\begin{tabular}{|c|c|c|c|c|c|c|}
\hline & $\begin{array}{c}\text { All } \\
\mathrm{n}=181\end{array}$ & $\begin{array}{c}\text { Q1 } \\
n=45\end{array}$ & $\begin{array}{c}\text { Q2 } \\
n=45\end{array}$ & $\begin{array}{c}\text { Q3 } \\
n=45\end{array}$ & $\begin{array}{c}\mathrm{Q} 4 \\
\mathrm{n}=46\end{array}$ & $\mathrm{p}$ \\
\hline Log (TG)/HDL-c & $0.064 \pm 0.031$ & $0.033 \pm 0.005$ & $0.048 \pm 0.004$ & $0.064 \pm 0.006$ & $0.108 \pm 0.025$ & - \\
\hline $\mathrm{TC}, \mathrm{mg} / \mathrm{dl}$ & $177 \pm 43$ & $188 \pm 40$ & $182 \pm 43$ & $178 \pm 37$ & $160 \pm 46$ & 0.010 \\
\hline LDL-c, mg/dl & $117 \pm 40$ & $116 \pm 37$ & $120 \pm 41$ & $120 \pm 38$ & $111 \pm 44$ & 0.673 \\
\hline HDL-c, mg/dl & $38 \pm 15$ & $57 \pm 10$ & $41 \pm 5$ & $33 \pm 4$ & $20 \pm 5$ & $<0.001$ \\
\hline Non HDL-c, mg/dl & $139 \pm 40$ & $131 \pm 38$ & $141 \pm 42$ & $146 \pm 36$ & $140 \pm 45$ & 0.387 \\
\hline $\mathrm{TG}, \mathrm{mg} / \mathrm{dl}$ & $100(45-50)$ & $77(45-139)$ & $100(45-236)$ & $115(45-650)$ & $116(45-414)$ & $<0.001$ \\
\hline Glycemia, mg/dl & $165(50-446)$ & $170(50-437)$ & $160(56-446)$ & $147(66-426)$ & $176(51-441)$ & 0.247 \\
\hline HbA1c, \% & $9.0(4.5-14.0)$ & $8.9(5.0-14.0)$ & $9.2(4.5-14.0)$ & $8.4(4.7-14.0)$ & $9.5(5.6-14.0)$ & 0.512 \\
\hline Creatinine, mg/dl & $1.1(0.4-33.6)$ & $1.1(0.4-26.5)$ & $1.1(0.6-6.0)$ & $1.2(0.6-4.8)$ & $1.2(0.5-33.6)$ & 0.257 \\
\hline GFR, MDRD, $\mathrm{ml} / \mathrm{min} / 1.73 \mathrm{~m}^{2}$ & $75(2-234)$ & $82(2-211)$ & $73(12-187)$ & $70(14-221)$ & $78(2-234)$ & 0.292 \\
\hline UAER, mg/g & $30(3-7800)$ & $42(4-7800)$ & $31(4-1600)$ & $17(3-2850)$ & $36(4-2000)$ & 0.365 \\
\hline
\end{tabular}

Data are expressed as mean \pm standard deviation, median and range or relative frequency in percent. Abbreviations: Log, logarithm; Q, quartile; TG, triglycerides; HDL-c, high-density lipoprotein-cholesterol; TC, total cholesterol; LDL-c, low-density lipoprotein-cholesterol; HbA1c, glycated hemoglobin; GFR, glomerular filtration rate; MDRD, modification of diet in renal disease; UAE, urinary albumin excretion.

Table 3. Atherogenic dyslipidemia and cardiovascular risk of the study population according to quartiles of Log (TG)/ HDL-c.

\begin{tabular}{|c|c|c|c|c|c|c|}
\hline & $\begin{array}{c}\text { All } \\
\mathrm{n}=181\end{array}$ & $\begin{array}{c}\mathrm{Q} 1 \\
\mathrm{n}=45\end{array}$ & $\begin{array}{c}\text { Q2 } \\
\mathrm{n}=45\end{array}$ & $\begin{array}{c}\text { Q3 } \\
\mathrm{n}=45\end{array}$ & $\begin{array}{c}\mathrm{Q} 4 \\
\mathrm{n}=46\end{array}$ & $\mathrm{p}$ \\
\hline Log (TG)/HDL-c & $0.064 \pm 0.031$ & $0.033 \pm 0.005$ & $0.048 \pm 0.004$ & $0.064 \pm 0.006$ & $0.108 \pm 0.025$ & - \\
\hline Hypertension, \% & 77 & 84 & 76 & 80 & 70 & 0.147 \\
\hline Global obesity, \% & 19 & 13 & 18 & 22 & 22 & 0.254 \\
\hline Abdominal obesity, \% & 54 & 56 & 49 & 58 & 54 & 0.874 \\
\hline $\mathrm{AD}, \%$ & 69 & 16 & 60 & 100 & 100 & $<0.001$ \\
\hline $\begin{array}{c}\mathrm{CV} \text { risk > 30\% } \\
\text { (WHO-LMIC), \% }\end{array}$ & 12.7 & 20.0 & 8.9 & 11.1 & 10.9 & 0.393 \\
\hline
\end{tabular}

Data are expressed as mean \pm standard deviation, median and range or relative frequency in percent. Abbreviations: Log, logarithm; Q, quartile; TG, triglycerides; HDL-c, high-density lipoprotein-cholesterol; HbA1c, glycated hemoglobin, TC, total cholesterol; CV, cardiovascular. 
microangiopathy, hypertension, increased cardiometabolic and cardiovascular risk. The racial difference in the suitability of Log (TG)/HDL-c ratio to predict cardiovascular risk is thought to rely upon the so-called "lipid or triglyceride" paradox in people of African descent [27]. If in Whites, the characteristic dyslipidemia follows the classic pattern of elevated TG and low HDL-c levels [15] [16], normal TG and low HDL-c levels characterize lipid profiles in African Americans, West Africans and black South Africans [31]-[33]. Therefore, screening tests such as Log (TG)/HDL-c ratio having TG as a key diagnostic criterion would not be appropriate in people of African descent [34]. Reasons for normal TG levels in Blacks and less predictive value of Log (TG)/HDL-c ratio in individuals of African descent is thought to rely upon the greater activity and less inhibition of lipoprotein lipase (LPL), the enzyme that clears TG-rich lipid particles from the circulation as well as the presence of hyperinsulinemia [27].

The mean levels of UAER did not increase across Log (TG)/HDL. In the general population, a recent study by Knight et al. [29] using the ROC curve to predict insulin resistance as assessed by HOMA-IR reported that Log (TG)/HDL-c failed to predict insulin resistance in obese women of African descent. Similar findings have been already reported by other authors such as Kim-Dorner et al. [35] and Summner et al. [31] [32] who found that Log (TG)/HDL-c ratio was acceptable marker of insulin resistance as assessed by fasting plasma insulin and HOMA-IR in Whites but poor predictors in Blacks. They concluded that this ratio would not be appropriate to predict insulin resistance in African Americans [28] [29]. Studies evaluating the relationship of Log (TG)/HDLc with insulin resistance in type 2 diabetes black Africans are not yet available to allow comparison with our findings.

The interpretation of the results of the present post-hoc analysis should take into account of some limitations. The major limitation of the present analysis was its derivation from a cross-sectional study and its post-hoc nature precluding temporal and causal relationship of Log (TG)/HDL-c with UAER and absolute cardiovascular risk. Direct measurement of insulin resistance was not performed in the present study. An additional limitation was the lack of repeated measurements of UAER and serum lipids, which are recommended to increase specificity.

\section{Conclusion}

Log (TG)/HDL-c as a surrogate estimate of atherogenic dyslipidemia failed to predict absolute cardiovascular risk in the present case series of black patients with type 2 diabetes, highlighting the need for tests adapted to people of African descent.

\section{Acknowledgements}

The authors gratefully thank Prof Dr Jean Robert Makulo Risassi for providing the database on urinary albumin excretion rate and lipid profile in type 2 diabetes patients. They also remain deeply indebted to Professor Bert Bamens of Katholiek Universiteit Leuven (KUL) for his invaluable contribution to the improvement of the quality of the present manuscript.

\section{Conflict of Interest}

None.

\section{Authors' Contribution}

KFM participated in protocol elaboration, data collection and analysis and reviewed the manuscript. LFB designed the study, participated in data analysis and wrote the manuscript.

MFIN contributed to the study design, performed statistical analysis and reviewed the manuscript. MJR provided database and reviewed the manuscript.

$\mathrm{BE}$ reviewed the manuscript.

NMN reviewed the manuscript.

\section{References}

[1] Naidoo, D.P. (2002) The Link between Microalbuminuria, Endothelial Dysfunction and Cardiovascular Disease in Diabetes. Cardiovasc J South Afr, 13, 194-199. 
[2] Hamilton, S.J. and Watts, G.F. (2013) Atherogenic Dyslipidemia and Combination Pharmacotherapy in Diabetes: Recent Trials. The Review of Diabetic Studies, 10, 191-203. http://dx.doi.org/10.1900/RDS.2013.10.191

[3] Agouridis, A.P., Rizos, C.V., Elisaf, M.S. and Filippatos, T.D. (2013) Does Combination Therapy with Statins and Fibrates Prevent Cardiovascular Disease in Diabetic Patients with Atherogenic Mixed Dyslipidemia? The Review of Diabetic Studies, 10, 171-190. http://dx.doi.org/10.1900/RDS.2013.10.171

[4] Pandeya, A., Sharma, M., Regmi, P., Basukala, A. and Lamsal, M.S. (2002) Pattern of Dyslipidemia and Evaluation of Non-HDL-c as a Marker of Risk Factor for Cardiovascular Disease in Type 2 Diabetes Mellitus. Nepal Med Coll J, 14, 278-282.

[5] American Diabetes Association: Standards of Medical Care in Diabetes-2009. Diabetes Care, 32, S13-S61. http://dx.doi.org/10.2337/dc09-S013

[6] Charlton-Menys, V., Betteridge, D.J., Colhoun, H., Fuller, J., France, M., Hitman, G.A., et al. (2009) Target of Statin Therapy; LDL Cholesterol, Non-HDL Cholesterol, and Apolipoprotein B in Type 2 Diabetes in the Collaborative Atorvastatin Diabetes Study (CRDS). Clinical Chemistry, 55, 473-480. http://dx.doi.org/10.1373/clinchem.2008.111401

[7] Wanner, C. and Krane, V. (2011) Recent Advances in the Treatment of Atherogenic Dyslipidemia in Type 2 Diabetes Mellitus. Kidney and Blood Pressure Research, 34, 209-217. http://dx.doi.org/10.1159/000326849

[8] Pang, J. and Chan, G.F. (2014) Origin and Therapy for Hypertriglyceridemia in Type 2 Diabetes. World Journal of Diabetes, 5, 165-175.

[9] Bosomworth, N.J. (2013) Approach to Identifying and Managing Atherogenic Dyslipidemia. A Metabolic Consequence of Obesity and Insulin Resistance. Canadian Family Physician, 59, 1169-1180.

[10] Ginsberg, H.N. and MacCallun, P.R. (2009) The Obesity, Metabolic Syndrome, and Type 2 Diabetes Mellitus Pandemic: II. Therapeutic Management of Atherogenic Dyslipidemia. Journal of Clinical Hypertension, 11, 520-527. http://dx.doi.org/10.1111/j.1559-4572.2009.00060.x

[11] de Boer, I.H., Astor, H.K., Kramer, H., Palmas, W., Rudser, K., Seliger, S., et al. (2008) Mild Elevations in Urine Albumin Excretion Are Associated with Atherogenic Lipid Abnormalities in the Multi-Ethnic Study of Atherosclerosis. Atherosclerosis, 197, 407-414. http://dx.doi.org/10.1016/j.atherosclerosis.2007.06.018

[12] Tseng, C.H. (2009) Differential Dyslipidemia with Albuminuria in Type 2 Diabetes Patients in Taiwan. Clinical Biochemistry, 42, 1019-1024. http://dx.doi.org/10.1016/j.clinbiochem.2009.03.013

[13] Tseng, C.H. (2005) Lipid Abnormalities Associated with Urinary Albumin Excretion Rate (UAER) in Type 2 Diabetic Patients. Kidney International, 67, 1547-1553. http://dx.doi.org/10.1111/j.1523-1755.2005.00235.X

[14] Tennebaum, A. and Fisman, E.Z. (2012) Fibrates Are an Essential Part of Modern Anti-Dyslipidemic Arsenal: Spotlight on Atherogenic Dyslipidemia and Residual Risk Reduction. Cardiovascular Diabetology, 11, 125. http://dx.doi.org/10.1186/1475-2840-11-125

[15] Hermans, P.H., Ahn, A.A. and Rousseau, M.F. (2012) The Atherogenic Dyslipidemia Ratio [Log(TG)/HDL-c] Is Associated with Residual Vascular Risk, Beta-Cell Function Loss and Microangiopathy in Type 2 Diabetes Females. Lipids in Health and Disease, 11, 132. http://dx.doi.org/10.1186/1476-511X-11-132

[16] Hermans, M.P., Ahn, S.A. and Rousseau, M.F. (2010) [log(TG)/HDL-c] Is Related to Both Residual Cardiometabolic Risk and $\beta$-Cell Function Loss in Type 2 Diabetes Males. Cardiovascular Diabetology, 9, 88. http://dx.doi.org/10.1186/1475-2840-9-88

[17] Onat, A., Can, G., Kaya, H. and Hergenç, G. (2010) Atherogenic Index of Plasma (Log10 Triglyceride/High-Density Lipoprotein-Cholesterol) Predicts High Blood Pressure, Diabetes, and Vascular Events. Journal of Clinical Lipidology, 4, 89-98. http://dx.doi.org/10.1016/j.jacl.2010.02.005

[18] Sirimarco, G., Labreuche, J., Bruckert, E., Goldstein, L.B., Fox, K.M., Rothwell, P.M., et al. (2014) Atherogenic Dyslipidemia and Residual Cardiovascular Risk in Statin-Treated Patients. Stroke, 45, 1429-1436. http://dx.doi.org/10.1161/STROKEAHA.113.004229

[19] Jisieike-Onuigbo, N.N., Unuigbe, E.I., Kalu, O.A., Oguejiofor, C.O. and Onuigbo, P.C. (2011) Prevalence of Dyslipidemia among Adult Diabetic Patients with Overt Diabetic Nephropathy in Anambra State South-East Nigeria. Nigerian Journal of Clinical Practice, 14, 171-175. http://dx.doi.org/10.4103/1119-3077.84009

[20] Risassi, J.R., Nseka, M.N., Mvitu, M., Muyer, M.T., Kimenyembo, W., Mandja, M., Bieleli, E.O., Mapatano, M.A., Lepira, F.B., Sumaili, E.K., Kaimbo, W.K., Nge, O., Buntinx, F. and Muls, E. (2010) Albuminuria during the Screening for Diabetes in a Semi Rural Area (Kisantu City, DR Congo). Nephrology \& Therapeutics, 6, 513-519.

[21] Bieleli, E. (1999) Study of Plasma Cholesterol and Lipoproteins among Adult Congelese Patients with Diabetes Mellitus. Thesis, School of Medicine, University of Kinshasa, Kinshasa. (In French)

[22] Bennett, P.H., Haffner, S., Kasiske, B.L., Keane, W.F., Mogensen, C.E., Parving, H.H., et al. (1995) Screening and 
Management of Microalbuminuria in Patients with Diabetes Mellitus: Recommendations to the Scientific Advisory Board of the National Kidney Foundation from an Ad Hoc Committee of the Council on Diabetes Mellitus of the National Kidney Foundation. American Journal of Kidney Diseases, 25, 107-112. http://dx.doi.org/10.1016/0272-6386(95)90636-3

[23] Friedelwald, W.T., Levy, R.I. and Fredrickson, D.S. (1972) Estimation of the Concentration of Low-Density Lipoprotein Cholesterol in Plasma, without Use of the Preparative Ultracentrifuge. Clinical Chemistry, 18, 499-502.

[24] Foro Dislipidemia Aterogénica (2013) Consenso multidisciplinar sobre dislipidemia aterogénica. Atherogenic Dyslipidemia: A Multidisciplinary Consensus Panel. Clínica e Investigación en Arteriosclerosis, 25, 83-91. http://dx.doi.org/10.1016/j.arteri.2013.03.001

[25] Mendis, S., Puska, P. and Norrving, B. (Eds.) (2011) Global Atlas on Cardiovascular Disease Prevention and Control. World Health Organization in Collaboration with the World Heart Federation and the World Stroke Organization, Geneva.

[26] Wang, S.H., Wang, L., Zhou, Y., Guo, Y.J., Yuan, Y., Li, F.F., et al. (2012) Prevalence and Control of Dyslipidaemia among Diabetic Patients with Microalbuminuria in a Chinese Hospital. Diabetes \& Vascular Disease Research, 10, 169-178. http://dx.doi.org/10.1177/1479164112454756

[27] Yu, S.S.K., Darleen, C., Castillo, D.C., Courville, A.B. and Sumner, A.E. (2012) The Triglyceride Paradox in People of African Descent. Metabolic Syndrome and Related Disorders, 10, 77-82. http://dx.doi.org/10.1089/met.2011.0108

[28] Nwagha, U.I., Ikekpeazu, E.J., Ejezie, F.E., Neboh, E.E. and Maduka, I.C. (2010) Atherogenic Index of Plasma as Auseful Predictor of Cardiovascular Risk among Postmenopausal Women in Enugu, Nigeria. African Health Sciences, 10, 248-252.

[29] Knight, M.G., Goedecke, J.H., Ricks, M., Evans, J., Levitt, N.S., Tulloch-Reid, M.K. and Sumner, A.E. (2011) The TG/HDL-C Ratio Does Not Predict Insulin Resistance in Overweight Women of African Descent: A Study of South African, African American and West African Women. Ethnicity \& Disease, 21, 490-494.

[30] Zoppini, G., Negri, C., Stoico, V., Casali, S., Pichiri, I. and Bonora, I. (2012) Triglyceride-High-Density Lipoprotein Is Associated with Microvascular Complications in Type 2 Diabetes Mellitus. Metabolism-Clinical and Experimental, 61, 22-29. http://dx.doi.org/10.1016/j.metabol.2011.05.004

[31] Sumner, A.E., Finley, K.B., Genovese, D.J., Criqui, M.H. and Boston, R.C. (2005) Fasting Triglyceride and the Triglyceride-HDL Cholesterol Ratio Are Not Markers of Insulin Resistance in African Americans. Archives of Internal Medicine, 165, 1395-1400. http://dx.doi.org/10.1001/archinte.165.12.1395

[32] Sumner, A.E., Harman, J.L., Buxbaum, S.G., Miller, B.V., Tambay, A.V., Wyatt, S.B., et al. (2010) The Triglyceride/High-Density Lipoprotein Cholesterol Ratio Fails to Predict Insulin Resistance in African-American Women: An Analysis of Jackson Heart Study. Metabolic Syndrome and Related Disorders, 8, 511-514. http://dx.doi.org/10.1089/met.2010.0028

[33] Goedecke, J.H., Utzschneider, K., Faulenbach, M.V., et al. (2010) Ethnic Differences in Serum Lipoproteins and Their Determinants in South African Women. Metabolism_Clinical and Experimental, 59, 1341-1350. http://dx.doi.org/10.1016/j.metabol.2009.12.018

[34] Osei, K. (2010) Metabolic Syndrome in Blacks: Are the Criteria Right? Current Diabetes Reports, 10, 199-208. http://dx.doi.org/10.1007/s11892-010-0116-4

[35] Kim-Dorner, S.J., Deuster, P.A., Zeno, S.A., Remaley, A.T. and Poth, M. (2010) Should Triglycerides and the Triglycerides to High-Density Lipoprotein Cholesterol Ratio Be Used as Surrogates for Insulin Resistance? MetabolismClinical and Experimental, 59, 299-304. http://dx.doi.org/10.1016/j.metabol.2009.07.027 